\title{
RELACION ENTRE EL ORIGEN FLORAL Y EL PERFIL DE ELEMENTOS MINERALES EN MIELES CHILENAS
}

\author{
RELATIONSHIP BETWEEN THE FLORAL ORIGINAND THE MINERAL PROFILE \\ IN CHILEAN HONEYS
}

\author{
Gloria Montenegro \& Carolina Fredes
}

\begin{abstract}
Laboratorio de Botánica, Departamento de Ciencias Vegetales, Facultad de Agronomía e Ingeniería Forestal, Pontificia Universidad Católica de Chile, Vicuña Mackenna 4860, Macul, Santiago, Chile. gmonten@puc.cl
\end{abstract}

\begin{abstract}
In this report, 61 honey samples were collected in beehives located between IV and X Administrative Region of Chile. We focussed the analysis in risky chemical elements for human health and in the main chemical elements found previously in Chilean honeys. The analysis of $\mathrm{Al}, \mathrm{Sr}, \mathrm{Cu}, \mathrm{Fe}, \mathrm{Mn}$ and $\mathrm{Zn}$ were done by wet acid digestion method by ICP-OES. Lotus pedunculatus and Eucryphia cordifolia honeys showed the highest Al contents related to their geographical origin (Bío-Bío and Los Lagos Regions). The principal component analysis did not show groups of honeys with the some floral origin and a typical chemical element profile.
\end{abstract}

Durante los últimos años, la denominación de origen y la inocuidad de los productos son los principales requerimientos del Mercado Internacional de la Miel. Por esta razón que certificar el origen floral y su procedencia constituye un gran desafío para el país (Danty 2004).

Si bien existen muchos antecedentes de la determinación de residuos químicos en mieles, producto del uso de acaricidas y antibióticos, la determinación de metales pesados y otros elementos traza en mieles no ha sido de gran investigación a nivel mundial (Fredes \& Montenegro 2006).

La presente nota informa la relación existente entre el origen floral de mieles y el perfil de seis elementos químicos, como: $\mathrm{Sr}, \mathrm{Al}, \mathrm{Fe}, \mathrm{Cu}, \mathrm{Mn}$ y Zn presentes en 61 mieles provenientes de colmenares ubicados entre la Región de Coquimbo y la de Los Lagos del país, recolectadas durante las temporadas 2002 y 2003.

La determinación del origen floral se realizó a través del análisis melisopalinológico mediante el método de Mauricio (1975), con un límite de confianza del $95 \%$. Mieles que presentaron un 50\% de participación de una misma especie fueron consideradas monoflorales.
La determinación de los elementos químicos se realizó mediante metodología de digestión ácida húmeda usando la adaptación de la metodología descrita por Devillers et al. (2002), utilizando espectrometría de emisión óptica de plasma inductivamente acoplado (ICP-OES). La preparación de estándares de calibración se realizó en solución de $\mathrm{HNO}_{3}$ al $2 \%$ al igual que las muestras de miel. Los estándares acuosos fueron preparados a partir de diluciones de una solución stock de Multielementos ICP ${ }^{\circledR}$ IV MERCK de concentración $1000 \mathrm{mg} \mathrm{l}^{-1}$, Merck. Todas las soluciones fueron preparadas gravimétricamente. Como control se utilizó un Material de Referencia Certificado para metales pesados MRC - CMQ I-004, Lote 006, elaborado en el Centro de Metrología Química - Fundación Chile.

Como miel control se utilizó una miel orgánica certificada, proveniente de PN Río Clarillo, Comuna Pirque, Reg. Metropolitana. Fecha de cosecha: enero de 2005. Origen: Monofloral de Quillaja saponaria Molina (75\% de quillay). Apicultor responsable: Bernhard Unland.

Para el análisis de los datos se realizó análisis de componentes principales (ACP) con las 61 muestras 
recolectadas. Las variables fueron los contenidos de los seis elementos químicos analizados. Se utilizó el programa Statgraphics para Windows 4.3 (Chen et al. 2000, Yu et al. 2001, Fredes \& Montenegro 2006).

En las muestras analizadas las especies predominantes en mieles de la zona central fueron Escallonia pulverulenta Pers. (siete camisas) y Quillaja saponaria (quillay), mientras que para la zona centro-sur y sur fueron Lotus pedunculatus Cav. (alfalfa chilota) y Eucryphia cordifolia Cav. (ulmo), tal como se observa en la Tabla I. Las especies presentes en mieles poliflorales fueron determinadas en frecuencias superiores al 5\%. Estos resultados coinciden con publicaciones anteriores que describen las principales mieles monoflorales endémicas de Chile (Montenegro 2002, Montenegro et al. 1989, 1992, 2003).

TABLA I. Composición floral de las mieles monoflorales y poliflorales estudiadas entre las regiones de Coquimbo y Los Lagos, Chile.

TABLE I. Floral composition of uniflora and multiflora honeys studied between Coquimbo and Los Lagos Regions, Chile.

\begin{tabular}{|c|c|c|}
\hline REGIÓN & ORIGEN FLORAL & ESPECIES PREDOMINANTES \\
\hline Coquimbo & $\begin{array}{l}\text { Monofloral } \\
\text { Polifloral }\end{array}$ & $\begin{array}{l}\text { No se identificaron } \\
\text { Brassica rapa L., Eucalyptus globulus Labill., Schinus latifolia Engl. } \\
\text { Medicago sativa L., Escallonia pulverulenta Pers. }\end{array}$ \\
\hline Valparaíso & $\begin{array}{l}\text { Monofloral } \\
\text { Polifloral }\end{array}$ & $\begin{array}{l}\text { Escallonia pulverulenta Pers., Quillaja saponaria Molina } \\
\text { Brassica rapa L., Rubus ulmifolius Schott, Eucalyptus globulus } \\
\text { Labill., Prunus domestica L., Lithrea caustica Hook. et Arn. }\end{array}$ \\
\hline Metropolitana & $\begin{array}{l}\text { Monofloral } \\
\text { Polifloral }\end{array}$ & $\begin{array}{l}\text { Quillaja saponaria Molina } \\
\text { Quillaja saponaria Molina, Rubus ulmifolius Schott, Lotus } \\
\text { pedunculatus Cav., Brassica rapa L., Escallonia rubra Pers. }\end{array}$ \\
\hline Libertador Bernardo O’Higgins & $\begin{array}{l}\text { Monofloral } \\
\text { Polifloral }\end{array}$ & $\begin{array}{l}\text { No se identificaron } \\
\text { Quillaja saponaria Molina, Rubus ulmifolius Schott, Escallonia } \\
\text { pulverulenta Pers., Medicago sativa L., Lotus pedunculatus Cav., } \\
\text { Eucalyptus globulus Labill., Luma apiculata (DC.) Burret }\end{array}$ \\
\hline Maule & $\begin{array}{l}\text { Monofloral } \\
\text { Polifloral }\end{array}$ & $\begin{array}{l}\text { Quillaja saponaria Molina } \\
\text { Rubus ulmifolius Schott, Quillaja saponaria Molina, Lotus } \\
\text { pedunculatus Cav., Luma apiculata (DC.) Burret, Fuchsia } \\
\text { magellanica Lam. }\end{array}$ \\
\hline Bío-Bío & $\begin{array}{l}\text { Monofloral } \\
\text { Polifloral }\end{array}$ & $\begin{array}{l}\text { No se identificaron. } \\
\text { Medicago sativa L., Echium vulgare L., Lotus pedunculatus Cav., } \\
\text { Gevuina avellana Molina, Rubus ulmifolius Schott, Medicago } \\
\text { polymorpha L., Luma apiculata (DC.) Burret }\end{array}$ \\
\hline Araucanía & $\begin{array}{l}\text { Monofloral } \\
\text { Polifloral }\end{array}$ & $\begin{array}{l}\text { Lotus pedunculatus Cav. } \\
\text { Lotus pedunculatus Cav., Trifolium pratense L., Eucryphia } \\
\text { cordifolia Cav., Weinmania trichosperma Cav. }\end{array}$ \\
\hline Los Lagos & $\begin{array}{l}\text { Monofloral } \\
\text { Polifloral }\end{array}$ & $\begin{array}{l}\text { Eucryphia cordifolia Cav. } \\
\text { Lotus pedunculatus Cav., Echium vulgare L., Luma apiculata } \\
\text { (DC.) Burret, Eucryphia cordifolia Cav., Rubus ulmifolius Schott, } \\
\text { Trifolium pratense L. }\end{array}$ \\
\hline
\end{tabular}


Los principales elementos encontrados en mieles en relación al resto de los elementos químicos analizados fueron Aluminio (Al) y estroncio ( $\mathrm{Sr}$ ), similar a los descritos por González et al. (2000) y Fredes \& Montenegro (2006). Las mieles de alfalfa chilota y ulmo presentaron los mayores contenidos de $\mathrm{Al}\left(3,2 \mathrm{mg} \mathrm{kg}^{-1}\right)$, lo que podría estar asociado al origen geográfico, ya que provienen de colmenares ubicados en suelos de origen volcánico distribuidos entre la Región del Bío-Bío y de Los Lagos con contenidos elevados de $\mathrm{Al}$ libre.

Los resultados del ACP indicaron tres componentes principales que explicaron el $73 \%$ de la variabilidad de los datos. A partir de los componentes principales 1 y 2 (CP1 y CP2) se realizó el mapa factorial CP1-CP2 que explicó el 58\% de la variabilidad total de los datos (40\% y $18 \%$, respectivamente), como se observa en la Figura 1.

La distribución a lo largo de CP1 sería principalmente dependiente de las concentraciones de Cu, Mn y Zn, considerados elementos esenciales o micronutrientes para las plantas superiores (Adriano 2001), mientras que la distribución a lo largo de CP2 sería principalmente dependiente de las concentraciones de $\mathrm{Al}$, considerado un elemento no esencial para las plantas (González 1994, Adriano 2001).

En la Figura 1 se observa un grupo principal de mieles cercanas al eje de intercepción de ambos componentes que se caracterizó por contenidos bajos de los elementos analizados. Por otro lado, dos mieles de alfalfa chilota, una de ulmo y tres mieles poliflorales presentaron los mayores contenidos de $\mathrm{Al}$ en un rango de 8,2 y $14,3 \mathrm{mg} \mathrm{kg}^{-1}$. En relación a las mieles con contenidos altos de micronutrientes, que se observan a lo largo de la gradiente de $\mathrm{CP} 1$, mieles poliflorales presentaron los mayores contenidos de $\mathrm{Cu}$, Mn y Zn (2,0; 3,1 y 4,7 $\mathrm{mg} \mathrm{kg}^{-1}$, respectivamente). Dentro del origen floral de estas mieles existió participación importante de plantas herbáceas.

Los resultados de este estudio no sustentan agrupaciones de mieles de un mismo origen floral con un perfil de elementos minerales similar. Sin embargo, sería interesante ampliar este tipo de estudios, analizando el contenido de elementos riesgosos como $\mathrm{Cd}, \mathrm{Pb}$ y $\mathrm{Cu}$ en las distintas partes de las estructuras reproductivas de las plantas que usan las abejas en zonas reconocidamente contaminadas.

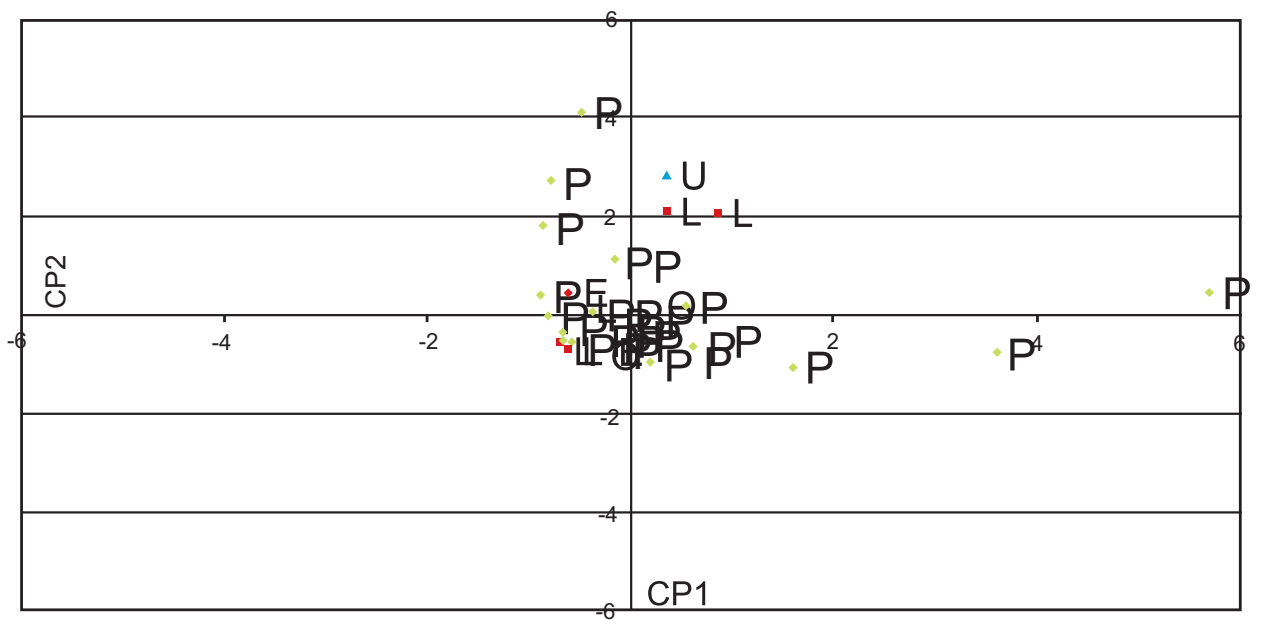

FIgURA 1. Mapa factorial CP1-CP2 para las 61 mieles analizadas (E: miel de Escallonia pulverulenta, Q: miel de Quillaja saponaria, L: miel de Lotus pedunculatus, U: miel de Eucryphia cordifolia, P: miel polifloral).

FIGURE 1. Factorial map (PC1-PC2) of 61 honeys analyzed (E: Escallonia pulverulenta honey, Q: Quillaja saponaria honey, L: Lotus pedunculatus honey, U: Eucryphia cordifolia honey, P: multiflora honey). 
TABLA II. Concentraciones promedio (y desviación estándar) de aluminio, estroncio, cobre, fierro, manganeso y zinc presentes en mieles de diferentes orígenes florales en $\mathrm{mg} \mathrm{kg}^{-1}$ (base a peso húmedo) en Chile. $\mathrm{N}=61$.

TABLE II. Concentrations mean (and standard deviation) of aluminium, strontium, copper, iron, manganese and zinc present in honey from different floral origins in $\mathrm{mg} \mathrm{kg}^{-1}$ (wet weight) in Chile. $\mathrm{N}=61$.

\begin{tabular}{lllllll}
\hline Origen floral & $\mathrm{Al}$ & $\mathrm{Sr}$ & $\mathrm{Cu}$ & $\mathrm{Fe}$ & $\mathrm{Mn}$ & $\mathrm{Zn}$ \\
\hline Escallonia pulverulenta & $1,5+0,3$ & $0,1+0,1$ & $0,0+0,0$ & $2,5+1,0$ & $0,3+0,3$ & $0,2+0,0$ \\
Quillaja saponaria & $1,0+0,8$ & $7,5+9,6$ & $0,0+0,0$ & $1,2+0,4$ & $0,5+0,3$ & $0,5+0,4$ \\
Lotus pedunculatus & $3,2+4,2$ & $0,0+0,0$ & $0,0+0,0$ & $1,3+1,5$ & $0,5+0,5$ & $1,2+1,5$ \\
Eucryphia cordifolia & $3,3+4,8$ & $0,0+0,0$ & $0,0+0,0$ & $0,9+1,3$ & $0,5+0,3$ & $0,9+1,3$ \\
Polifloral & $1,7+2,6$ & $1,8+4,2$ & $0,1+0,3$ & $1,6+2,2$ & $0,6+0,7$ & $0,7+0,9$
\end{tabular}

\section{AGRADECIMIENTOS}

A Rodrigo Pizarro por la determinación del origen floral de las mieles estudiadas. Al proyecto FONDECYT 1060535.

\section{BIBLIOGRAFIA}

Adriano, D.C. 2001. Trace elements in terrestrial environments. Biogeochemistry, bioavailability and risk of metals. Second Edition. Springer-Verlag. $867 \mathrm{pp}$.

Chen, C.Y., R.S. Stemberger, B. Klaue, J.D. Blum, P.C. Pickhardt \& C.L. Folt. 2000. Accumulation of heavy metals in food web components across a gradient of lakes. Limnology and Oceanography. 45(7):1525-1536.

DAnTy, J. 2004. El mercado de la miel. ODEPA Chile. $11 \mathrm{pp}$.

Devillers, J., J. Dore, M. Marenco, F. Poirier-Duchene, N. GALAND \& C. VIEL. 2002. Chemometrical analysis of 18 metallic and nonmetallic elements found in honey sold in France. Journal of Agricultural and Food Chemistry. 50 (21): 5998-6007.

Fredes, C. \& G. Montenegro. 2006. Contenidos de metales pesados y otros elementos traza en mieles de abejas en Chile. Revista Ciencia e Investigación Agraria 33(1): 57-66.

GonZÁLEZ S. 1994. Geoquímica de metales pesados en Chile. Libro de resúmenes. VI Simposio sobre contaminación ambiental. 19-20 de abril, Universidad de Chile. 10-44.

GonzÁlez, A., J. Gómez, R. García-Villanova, T. Rivas, R. Ardanuy \& J. Sanchez. 2000. Geographical discrimination of honeys by using mineral composition and common chemical quality parameters. Journal of the Science of Food and Agriculture 80: 157-165.

Mauricio A. 1975. Microscopy of honey. In: E. Crane (ed.), Honey: a comprehensive survey. London, England. pp: 240-257.

Montenegro, G., M. Schuck, A.M. Mujica \& S. Teillier. 1989. Flora utilizada por abejas melíferas (Apis mellifera) como fuente de polen en Paine, Región Metropolitana, Chile. Revista Ciencia e Investigación Agraria. 16(1-2): 47-53.

Montenegro G., M.Gómez \& G. Avila. 1992. Importancia relativa de especies cuyo polen es utilizado por Apis mellifera en el área de la Reserva Nacional Los Ruiles, VII Región de Chile. Acta Botánica Malacitana.17:167-174.

Montenegro G. 2002. Chile, nuestra flora útil. Universidad Católica de Chile. Chile. 267 pp.

Montenegro, G., R. Pizarro, G. Avila, R. Castro, C. Ríos, O. MuÑoz, F. BAs \& M. Gómez. 2003. Origen botánico y propiedades químicas de las mieles de la Región Mediterránea Árida de Chile. Revista Ciencia e Investigación Agraria. 30(3):161-174.

Yu, K.C., L.J. Tsai, S.H. Chen, D.J. Chang \& S.T. Ho. 2001. Multivariate correlations of geochemical binding phases if heavy metals in contaminated river sediment. Journal of Environmental Science and Health. A36(1):1-16. 